\title{
The Deterministic Version of the Glimm Scheme*
}

\section{Tai-Ping Liu ${ }^{\star \star}$}

Department of Mathematics, University of Maryland, College Park, Maryland 20742, USA

\begin{abstract}
The Glimm scheme for solving hyperbolic conservation laws has a stochastic feature; it depends on a random sequence. The purpose of this paper is to show that the scheme converges for any equidistributed sequence. Thus the scheme becomes deterministic.
\end{abstract}

\section{Introduction}

We consider the initial value problem for the system of general conservation laws:

$$
\begin{aligned}
& \frac{\partial u}{\partial t}+\frac{\partial f(u)}{\partial x}=0, \quad t \geqq 0, \quad-\infty<x<\infty, \\
& u(x, 0)=u_{0}(x), \quad-\infty<x<\infty,
\end{aligned}
$$

where $u$ and $f(u)$ are $n$-vectors, and $f$ is a smooth function of $u$. The system is assumed to be strictly hyperbolic, that is, the matrix $\partial f(u) / \partial u$ has real and distinct eigenvalues $\lambda_{1}(u)<\lambda_{2}(u)<\ldots<\lambda_{n}(u)$ with corresponding right eigenvectors $r_{1}(u)$, $r_{2}(u), \ldots, r_{n}(u)$. Since $(1.1),(1.2)$ in general does not have smooth solution; we look for weak solution in the distributional sense. A bounded measurable function $u(x, t)$ is a weak solution if

$$
\int_{t \geqq 0}\left[u \frac{\partial \varphi}{\partial t}+f(u) \frac{\partial \varphi}{\partial x}\right] d x d t+\int_{t=0} u_{0}(x) \varphi(x, 0)=0
$$

for any smooth function $\varphi(x, t)$ with compact support in $t \geqq 0$.

In [1], Glimm introduces a difference scheme for solving (1.1), (1.2). We now describe briefly the Glimm scheme. Choose any mesh lengths $r, s, r / s$ bounded, which satisfy the Courant-Friedrich-Lewy condition:

$$
\frac{r}{S} \geqq \max _{i=1,2, \ldots, n}\left|\lambda_{i}(u)\right|
$$

* Results obtained at the Courant Institute of Mathematical Sciences, New York University while the author was a Visiting Member at the Institute; this work was supported by the National Science Foundation, Grant NSF-MCS 76-07039

$\star \star$ On leave from the University of Maryland, College Park 
for any $u$ under consideration. The approximate solution $u_{r}\left(x, t ; a_{m}\right)$ is constructed inductively according to a prechosen sequence $\left\{a_{m}\right\}_{m=1}^{\infty},-1<a_{m}<1$, in the following way. Set $u_{r}\left(x, 0 ; a_{m}\right)=u_{0}(i r), i$ odd, for $(i-1) r<x<(i+1) r$. Suppose that $u_{r}\left(x, t ; a_{m}\right)$ is defined for $t<j s$, then we set

$$
u_{r}\left(x, j s ; a_{m}\right)=u_{r}\left(\left(i+1+a_{j}\right) r-0, j s-0\right), \quad i r<x<(i+2) r .
$$

for any $i=0, \pm 1, \pm 2, \ldots$, with $i+j$ even. Thus $u_{r}\left(x, j s ; a_{m}\right)$ is a step function of $x$ with possible discontinuity at $(i r, j s), i+j$ even. We then define $u_{r}\left(x, t, a_{m}\right) . j s<t<(j+1) s$, by resolving these discontinuities, so that in the zone $j s<t<(j+1) s$ the approximate solution is exact and consists of elementary waves issued from $(i r, j s), i+j$ even.

Here we use Lax's solution [3] of (1.1)-(1.2) in the case when the initial data has a single jump discontinuity. This problem is known as the Riemann problem and it is sufficient to extend the solution forward by one time step, because of the CourantFriedrick-Lewry condition. In fact the waves in the solution of the Riemann problem do not propagate faster than the local sound speeds $\lambda_{i}$, and thus cannot interact within one time step. However the solution so constructed at one time step forward is no longer piecewise constant, and the role of (1.4) is an approximation to restore this property. Thus in summary, the scheme pieces together solutions [3] of Riemann problems to obtain an approximate solution for a general class of data (restricted however to be nearly constant).

Glimm introduces a nonlinear functional to take care of non-linear wave interactions. He shows that when the system (1.1) is genuinely nonlinear (cf. Lax [3]) and the initial data have sufficiently small total variation, then there exists a constant independent of $t, r$, and $\left\{a_{m}\right\}$ such that

$$
\text { T.V. } u_{r}\left(x, t ; a_{m}\right) \leqq \text { const T.V. } u_{0}(x),
$$

where T.V. denotes the total variation over $x \in(-\infty, \infty)$. It follows by a compactness argument based on the Helly's theorem that the approximate solutions converge to a function as $r$ goes to zero. For the limiting function to be an exact solution, it must satisfies (1.3). Since $u_{r}$ is not exact along $t=j s, j=0,1,2, \ldots$, simple calculation yields:

$$
\begin{gathered}
\iint_{t \geqq 0} u_{r} \frac{\partial \varphi}{\partial t}+f\left(u_{r}\right) \frac{\partial \varphi}{\partial t} d x d t+\int_{t=0} u_{r} \varphi d x=E\left(R, \varphi, a_{m}\right) \equiv \sum_{j=0}^{\infty} E_{j}\left(r, \varphi, a_{m}\right), \\
E_{j}\left(r, \varphi, a_{m}\right) \equiv \int_{-\infty}^{\infty} u_{r}\left(x, j s+0 ; a_{m}\right)-u_{r}\left(x, j s-0 ; a_{m}\right) \varphi(x, j s) d t
\end{gathered}
$$

By showing that $\left\|E\left(r, \varphi, a_{m}\right)\right\|_{L^{2}(A)} \rightarrow 0$ as $r \rightarrow 0$, Glimm concludes that for almost all $\left\{a_{m}\right\}$ in $A$ the limit function is an exact solution. Here $A$ is the product of countable copies of interval $(-1,1)$ with the usual topology.

Our main purpose is to identify the sequences $\left\{a_{m}\right\}$ which assure that the limit of approximate solutions is an exact solution. We show that it is sufficient for the sequence $\left\{a_{m}\right\}$ to be equidistributed in $(-1,1)$ (Theorem 3.1), i.e.

$$
\lim _{k \rightarrow \infty} \frac{B\left(a_{m}, k, I\right)}{k}=\frac{\mu(I)}{2}
$$


for any subinterval $I$ of $(-1,1)$. Here $B\left(a_{m}, k, I\right)$ denotes the number of $m, 1 \leqq m \leqq k$, with $a_{m} \in I$, and $\mu(I)$ is the length of $I$. We now show by a simple example the necessity of this condition.

Consider a solution which is a discontinuity along $x-c t=0$ connecting $u_{1}$ on the left and $u_{2}$ on the right. The approximate solution is exact for $0 \leqq t<s$. For $s<t<2 s$, the approximate solution consists of a discontinuity issued from $(-\mathrm{r}, \mathrm{s})$ or $(r, s)$ respectively depending on whether $a_{1} r>c s$ or $a_{1} r \leqq c s$. Inductively, we find that for $t$ between $j s$ and $(j+1) s$, the approximate solution consists of a discontinuity issued from $(i r, j s)$ where

$$
\operatorname{ir}=r B\left(a_{m}, i,(-1, c s / r)\right)-r\left[i-B\left(a_{m}, i,(-1, c s / r)\right)\right] .
$$

On the other hand the exact solution has a discontinuity at $(c j s, j s)$. Thus in order for the approximate solution to approach the exact solution, we need $i r \rightarrow c j s$ as $j \rightarrow \infty$ and $r \rightarrow 0$, or, equivalently, (1.7) holds for $I=(-1, \alpha), \alpha=c s / r$. Since the nonlinear wave speed $c=\lambda_{i}(u)$ may assume arbitrary values, we see that (1.7) must hold for arbitrary $I$ and the sequence $\left\{a_{m}\right\}$ is equidistributed.

In the above example we need only to calculate the speed of one wave which is constant. For general solutions, waves may change speed and strength due to nonlinear interactions and cancellations. To minimize these effects, we take advantage of the fact that for any equidistributed sequence $\left\{a_{m}\right\}$, the subsequence $\left\{a_{m} \mid q N \leqq m \leqq p N\right\}$ is also equidistributed for any fixed $p>p \geqq 0$ as $N \rightarrow \infty$ [cf. (3.1) and (3.14)]. Our main assumption is that the total amount of interactions and cancellations is finite. This is the case either for initial data with sufficiently small total variation as noted by Glimm-Lax [2], or for special systems (cf. Liu [5]). In Glimm [1], the system (1.1) is assumed to be genuinely-nonlinear in the sense of Lax [3] where the Riemann problem is solved and analysized. Since the Riemann problem for general system has been solved by Liu [4] by introducing a general entropy condition, we do not have to assume that the system is genuinely nonlinear. Nevertheless, for simplicity, we will carry out our analysis only when the system (1.1) is genuinely nonlinear and the initial data (1.2) have small total variation.

Our method depends on the detailed analysis of wave interactions and cancellations which is described in Section 2. It is shown that although the change of wave strength due to interaction is second order, Theorems 2.1,2.2, the change of the wave speed is inversely proportional to the wave strength, Theorem 2.3 . The main theorem, Theorem 3.1, is proved in Section 3 where we devise an elaborate induction process to partition the elementary waves into subwaves so that the evolution of the speed and strength of these subwaves can be traced (Lemma 3.2).

For a treatment of the general theory of conservation laws, the interested reader may consult the excellent survey article of Lax [6].

\section{Estimates}

We will assume for simplicity that system (1.1) is genuinely nonlinear, i.e. $r_{i}(u)$ $\cdot \nabla \lambda_{i}(u) \neq 0, i=1,2, \ldots, n$, for all $u$ and the initial data (1.2) have small total variation. The rarefaction curve $R_{i}(u)$ is the integral curve of $r_{i}(u)$ through $u_{0}$ and the shock curve $S_{i}(u)$ is the Rankine-Hugoniot curve which is tangent to $R_{i}(u)$ at $u_{0}$, i.e. for any $u \in S_{i}\left(u_{0}\right)$,

$$
\left(u-u_{0}\right) \sigma\left(u, u_{0}\right)=f(u)-f\left(u_{0}\right)
$$


for some scalar $\sigma\left(u, u_{0}\right)$, the shock speed, which satisfies

$$
\lim _{u \rightarrow u_{0}} \sigma\left(u, u_{0}\right)=\lambda_{i}\left(u_{0}\right) .
$$

The shock curve $S_{i}\left(u_{0}\right)$ exists at least in a small neighborhood of $u_{0}$, Lax [3]. These curves, are divided into

$$
\begin{aligned}
& R_{i}^{ \pm}\left(u_{0}\right)=\left\{u \in R_{i}\left(u_{0}\right) \mid \lambda_{i}(u) \gtrless \lambda_{i}\left(u_{0}\right)\right\}, \\
& S_{i}^{ \pm}\left(u_{0}\right)=\left\{u \in S_{i}\left(u_{0}\right) \mid \sigma\left(u, u_{0}\right) \gtrless \lambda_{i}\left(u_{0}\right)\right\},
\end{aligned}
$$

so that $u$ can be connected to $u_{0}$ on the left by an $i$-rarefaction (or $i$-shock) wave if $u \in R^{+}\left(u_{0}\right)$ [or $\left.u \in S^{-}\left(u_{0}\right)\right]$. The shock wave $\left(u, u_{0}\right), u \in S_{i}^{-}\left(u_{0}\right)$ is stable in the sense of Lax $:\left(u_{0}\right)>\sigma\left(u, u_{0}\right)>\lambda(u)$. The Riemann problem $\left(u_{e}, u_{r}\right)$ is an initial value problem for (1.1) with two constant initial states:

$$
u(x, 0)=\left\{\begin{array}{lll}
u_{e} & \text { for } & x<0 \\
u_{r} & \text { for } & x>0
\end{array} .\right.
$$

To solve the Riemann problem $\left(u_{e}, u_{r}\right)$, we find vectors $u_{i}, i=0,2, \ldots, n, u_{0}=u_{e}$, $u_{n}=u_{r}, u_{i} \in S_{i}^{-}\left(u_{i-1}\right) \cup R^{+}\left(u_{i-1}\right)$, so that the solution consists of elementary $i$-waves $\left(u_{i-1}, u_{i}\right)$. Given any parameter $\mu_{i}$ along $S_{i}^{-} \cup R_{i}^{+}$, we set the strength of the $i$-waves in $\left(u_{e}, u_{r}\right)$ as:

$$
\left(u_{e}, u_{r}\right)=\mu_{i}\left(u_{i}\right)-\mu_{i}\left(u_{i-1}\right) .
$$

We always choose $\mu_{i}$ so that shock waves have negative strengths and rarefaction waves have positive strengths. Sometimes it is convenient to set $\mu_{i}=\lambda_{i}$. The following theorem on wave interactions is due to Glimm.

Theorem 2.1. For any nearby states $u_{e}, u_{m}, u_{r}$, there exists bounds $0(1)$ depending only on the system (1.1) so that

$$
\left(u_{e}, u_{r}\right)_{i}=\left(u_{e}, u_{m}\right)_{i}+\left(u_{m}, u_{r}\right)_{i}+0(1) Q\left(u_{e}, u_{m}, u_{r}\right),
$$

where $Q$ measures the potential amount of interaction and is defined as follows:

$A j$-wave on the left interacts a $k$-wave on the right if either $j>k, o r, j=k$ and at least one of the waves is a shock wave. We set

$$
Q\left(u_{e}, u_{m}, u_{r}\right)=\sum\left(u_{e}, u_{m}\right)_{j}\left(u_{m}, u_{r}\right)_{k}
$$

the summation being over all interacting waves.

The above Theorem is proved by the implicit function theorem, [1]. The following theorem gives a more detailed description of wave interactions.

Theorem 2.2. Suppose that $u_{e}, u_{r}=v_{e}$, and $v_{r}$ are nearby states and the Riemann problems $\left(u_{e}, u_{r}\right),\left(v_{e}, v_{r}\right)$, and $\left(u_{e}, v_{r}\right)$ are solved respectively by $i$-waves $\left(u_{i-1}, u_{i}\right)$, $\left(v_{i-1}, v_{i}\right)$, and $\left(w_{i-1}, w_{i}\right), i=0,1, \ldots, n$. We set $\alpha_{i}=u_{i}-u_{i-1}, \beta_{i}=v_{i}-v_{i-1}$ and $\gamma_{i}=w_{i}$ $-w_{i-1}$. Then

$$
\begin{aligned}
w_{i} & =v_{i}-\alpha_{n}-\alpha_{n-1}-\ldots-\alpha_{i+1}+0(1) Q\left(u_{e}, u_{r}, v_{r}\right) \\
& =u_{i}+\beta_{1}+\beta_{2}+\ldots+\beta_{i}+0(1) Q\left(u_{e}, u_{r}, v_{r}\right), \\
\gamma_{i} & =\alpha_{i}+\beta_{i}+0(1) Q\left(u_{e}, u_{r}, v_{r}\right) .
\end{aligned}
$$


Proof. We note that (2.3) is equivalent to Theorem 2.1 and is an easy consequence of (2.2). Estimate (2.2) is proved by the implicit function theorem. For brevity, we will use Theorem 2.1 to prove (2.2) by induction. Suppose that (2.2) holds when $u_{0}=u_{1}$ $=\ldots=u_{p}, p \in\{1,2, \ldots, n\}$. This is obviously the case when $p=n$. We have to show that it also holds when $u_{0}=u_{1}=\ldots=u_{p-1}$ and $u_{p-1}$ may not equal $u_{p}$. Denote by $\left(\tilde{w}_{i-1}, \tilde{w}_{i}\right), i=1,2, \ldots, n$, the elementary waves which solve the Riemann problem $\left(u_{p}, v_{r}\right)$. By induction hypothesis, we have

$$
\begin{aligned}
\tilde{w}_{i} & =v_{i}-\alpha_{n}-\alpha_{n-1}-\ldots-\alpha_{p}+0(1) Q\left(u_{p}, u_{r}, v_{r}\right) \\
& =u_{p}+\beta_{1}+\beta_{2}+\ldots+\beta_{i}+0(1) Q\left(u_{p}, u_{r}, v_{r}\right), \text { for } i<p,
\end{aligned}
$$

and

$$
\begin{aligned}
\tilde{w}_{i} & =v_{i}-\alpha_{n}-\alpha_{n-1}-\ldots-\alpha_{i+1}+0(1) Q\left(u_{p}, u_{r}, v_{r}\right) \\
& =u_{i}+\beta_{1}+\beta_{2}+\ldots+\beta_{i}+0(1) Q\left(u_{p}, u_{r}, v_{r}\right), \text { for } i \geqq p .
\end{aligned}
$$

We note that $\left(u_{p-1}, u_{p}\right)$ interacts with $\left(\tilde{w}_{i-1}, \tilde{w}_{i}\right), i=1,2, \ldots, n$ to produce $\left(w_{i-1}, w_{i}\right)$, $i=1,2, \ldots, n$. Thus it follows from Theorem 2.1 that for $i \neq p$

$$
\gamma_{i}=\tilde{w}_{i}-\tilde{w}_{i-1}+O(1) Q\left(u_{p-1}, u_{p}, v_{r}\right) .
$$

It follows from the definition of $Q$ and Theorem 2.1 that $Q\left(u_{p-1}, u_{p}, v_{r}\right) \leqq Q\left(u_{p-1}, u_{r}\right.$, $\left.v_{r}\right)=Q\left(u_{e}, u_{r}, v_{r}\right)$, and so

$$
\gamma_{i}=\tilde{w}_{i}-\tilde{w}_{i-1}+0(1) Q\left(u_{e}, u_{r}, v_{r}\right) \text {. }
$$

Since $w_{0}=u_{p-1}$ and $w_{n}=v_{r}$, we conclude from (2.5) and (2.6) that for $i \geqq p$

$$
\begin{aligned}
w_{i} & =-\sum_{k=i+1}^{n}\left(w_{k}-w_{k-1}\right)+u_{n} \\
& =-\sum_{k=i+1}^{n}\left(\tilde{w}_{k}-\tilde{w}_{k-1}\right)+u_{n}+O(1) Q\left(u_{e}, u_{r}, v_{r}\right) \\
& =-\sum_{k=i+1}^{n}\left(u_{k}-u_{k-1}+\beta_{k}-\beta_{k-1}\right)+u_{n}+O(1) Q\left(u_{p}, u_{r}, v_{r}\right) \\
& =u_{i}+\beta_{1}+\beta_{2}+\ldots+\beta_{i}+0(1) Q\left(u_{p}, u_{r}, v_{r}\right) .
\end{aligned}
$$

This proves the second part of (2.2) for $i \geqq p$. Other cases are proved using (2.4) (2.6) in a similar way. Q.E.D.

The following theorem describes how wave speeds change due to interactions and cancellations.

Theorem 2.3. Assume that same hypothesis as in Theorem 2.2 hold. When $\left(u_{i-i}, u_{i}\right)$ is a shock wave (or a rarefaction wave) we write $\alpha_{i} \equiv u_{i}-u_{i-1}<0$ ( or $\alpha_{i} \geqq 0$ ) etc. We have

(i) if $\alpha_{i} \leqq 0$ and $\beta_{i} \leqq 0$, then either $\left\|\gamma_{i}\right\|=0(1) Q\left(u_{e}, u_{r}, v_{r}\right)$ or $\gamma_{i} \leqq 0$ and $\left|\sigma\left(w_{i-1}, w_{i}\right)-\sigma\left(u_{i-1}, u_{i}\right)\right| \cdot\left\|\alpha_{i}\right\|+\left|\sigma\left(w_{i-1}, w_{i}\right)-\sigma\left(v_{i-1}, v_{i}\right)\right| \cdot\left\|\beta_{i}\right\|=0(1) Q\left(u_{e}, u_{r}, v_{r}\right)$,

(ii) if $\alpha_{i} \geqq 0$ and $\beta_{i} \geqq 0$, then either $\left\|\gamma_{i}\right\|=0(1) Q\left(u_{e}, u_{r}, v_{r}\right)$ or $\gamma_{i} \geqq 0$ and $\left|\lambda_{i}\left(u_{i-1}\right)-\lambda_{i}\left(w_{i-1}\right)\right| \cdot\left\|\alpha_{i}\right\|+\left|\lambda_{i}\left(v_{i}\right)-\lambda_{i}\left(w_{i}\right)\right| \cdot\left\|\beta_{i}\right\|=0(1) Q\left(u_{e}, u_{r}, v_{r}\right)$, 
(iii) if $\alpha_{i}<0, \beta_{i} \geqq 0$, and $\gamma_{i} \geqq 0$, then

$\left|\lambda_{i}\left(w_{i}\right)-\lambda_{i}\left(v_{i}\right)\right| \cdot\left\|\beta_{i}\right\|=0(1) Q\left(u_{e}, u_{r}, v_{r}\right)$,

(iv) if $\alpha_{i}<0, \beta_{i} \geqq 0$, and $\gamma_{i}<0$, then

$\left|\sigma\left(w_{i-1}, w_{i}\right)-\sigma\left(u_{i-1}, u_{i}\right)\right| \cdot\left\|\alpha_{i}\right\|=0(1) Q\left(u_{e}, u_{r}, v_{r}\right)$,

(v) if $\alpha_{i} \geqq 0, \beta_{i}<0$, and $\gamma_{i} \geqq 0$, then

$\left|\lambda_{i}\left(w_{i-1}\right)-\lambda_{i}\left(u_{i-1}\right)\right| \cdot\left\|\alpha_{i}\right\|=0(1) Q\left(u_{e}, u_{r}, v_{r}\right)$,

(iv) if $\alpha_{i} \geqq 0, \beta_{i}<0$, and $\gamma_{i}<0$, then

$\left|\sigma\left(v_{i-1}, v_{i}\right)-\sigma\left(w_{i-1}, w_{i}\right)\right| \cdot\left\|\beta_{i}\right\|=0(1) Q\left(u_{e}, u_{r}, v_{r}\right)$.

Proof. We will only prove (i), (ii), and (iii); other cases are proved similarly. Since the shock speed depends continuously on its states, we have from Theorem 2.2 that in the case (i),

$$
\begin{aligned}
& \left|\sigma\left(w_{i-1}, w_{i}\right)-\sigma\left(u_{i-1}, u_{i}\right)\right| \cdot\left\|\alpha_{i}\right\| \\
& =0(1)\left(\left\|w_{i-1}-u_{i-1}\right\|+\left\|w_{i}-u_{i}\right\|\right) \cdot\left\|\alpha_{i}\right\| \\
& =0(1)\left(\left\|2 \beta_{1}+\ldots+2 \beta_{i-1}\right\|+\left\|\beta_{i}\right\|\right) \cdot\left\|\alpha_{i}\right\|
\end{aligned}
$$

which is $0(1) Q\left(u_{e}, u_{r}, v_{r}\right)$ according to the definition of $Q$. The second part of (i) is proved similarly. Similarly in case (ii) we have

$$
\begin{aligned}
& \left|\lambda_{i}\left(u_{i-1}\right)-\lambda\left(w_{i-1}\right)\right|\left\|\alpha_{i}\right\| \\
& =0(1)\left\|u_{i-1}-w_{i-1}\right\|\left\|\alpha_{i}\right\| \\
& =0(1)\left\|\beta_{1}+\ldots+\beta_{i-1}\right\|\left\|\alpha_{i}\right\|+0(1) Q\left(u_{e}, u_{r}, v_{r}\right) \\
& =0(1) Q\left(u_{e}, u_{r}, v_{r}\right) .
\end{aligned}
$$

In case (iii) we have

$$
\begin{aligned}
& \left|\lambda_{i}\left(w_{i}\right)-\lambda_{i}\left(v_{i}\right)\right|\left\|\beta_{i}\right\| \\
& =0(1)\left\|w_{i}-v_{i}\right\|\left\|\beta_{i}\right\| \\
& =0(1)\left\|\alpha_{n}+\alpha_{n-1}+\ldots+\alpha_{i+1}\right\|\left\|\beta_{i}\right\|+0(1) Q\left(u_{e}, u_{r}, v_{r}\right) \\
& =0(1) Q\left(u_{e}, u_{r}, v_{r}\right) . \quad \text { Q.E.D. }
\end{aligned}
$$

The following lemma will be used later for the partition of waves and is an easy consequence of the continuous dependence of $R_{i}\left(u_{0}\right)$ and $S_{i}\left(u_{0}\right)$ on $u_{0}$. The proof is omitted.

Lemma 2.4. Suppose that all states are close to each other. Then

(i) if $w_{j} \in R_{i}\left(w_{0}\right), u_{j} \in R_{i}\left(u_{0}\right), j=1,2$, and $\lambda_{i}\left(w_{j}\right)-\lambda_{i}\left(w_{0}\right)=\lambda_{i}\left(u_{j}\right)-\lambda_{i}\left(u_{0}\right), j=1,2$, then

$$
\begin{aligned}
w_{1}-w_{2} & =u_{1}-u_{2}+0(1)\left\|w_{0}-u_{0}\right\|\left\|w_{1}-w_{2}\right\| \\
& =u_{1}-u_{2}+0(1)\left\|w_{0}-u_{0}\right\|\left\|u_{1}-u_{2}\right\|,
\end{aligned}
$$

(ii) if $w_{j} \in S_{i}\left(w_{0}\right), j=1,2, u_{1} \in S_{i}\left(u_{0}\right)$ and $\lambda_{i}\left(w_{2}\right)-\lambda_{i}\left(w_{1}\right)=\lambda_{i}\left(u_{1}\right)-\lambda_{i}\left(u_{0}\right)$, then

$$
\begin{aligned}
w_{1}-w_{2} & =u_{0}-u_{1}+0(1)\left(\left\|u_{0}-w_{0}\right\|+\left\|w_{0}-w_{1}\right\|\right)\left\|u_{1}-u_{0}\right\| \\
& =u_{0}-u_{1}+0(1)\left(\left\|u_{0}-w_{0}\right\|+\left\|w_{0}-w_{1}\right\|\right)\left\|w_{2}-w_{1}\right\| .
\end{aligned}
$$


For any given sequence $\left\{a_{m}\right\}$, the xt-plane consists of diamonds $\Delta_{i, j}$ with center $(i r, j s)$ and vertices $\left(\left(i+a_{j-1}\right) r,(j-1) s\right), \quad\left(\left(i-1+a_{j}\right) r, j s\right), \quad\left(\left(i+1+a_{j}\right) r, j s\right)$, and $\left(\left(i+a_{j+1}\right) r,(j+1) s\right)$. An $I$-curve is a space-like curve connecting vertices of the above form. An I-curve $J_{2}$ is an immediate successor of $I$-curve $J_{1}$ if $J_{1}$ and $J_{2}$ pass through same vertices except two and $J_{2}$ lies toward larger than $J_{1}$. The waves entering each diamond $\Delta$ are solutions of two Riemann problems, say, $\left(u_{e}, u_{m}\right)$ and $\left(u_{m}, u_{r}\right)$. We denote by $Q(\Delta) \equiv Q\left(u_{e}, u_{m}, u_{r}\right)$ the amount of interaction in $\Delta$ and $C_{i}(\Delta)=\frac{1}{2}\left(\left|\left(u_{e}, u_{m}\right)_{i}\right|\right.$ $\left.+\left|\left(u_{m}, u_{r}\right)_{i}\right|-\left|\left(u_{e}, u_{m}\right)_{i}+\left(u_{m}, u_{r}\right)_{i}\right|\right)$ the amount of cancellation in $\Delta$. Estimate $(2.1)$ can be rewritten as:

$$
\left|\left(u_{e}, u_{m}\right)_{i}\right|=\left|\left(u_{e}, u_{m}\right)_{i}\right|+\left|\left(u_{m}, u_{r}\right)_{i}\right|+C_{i}(\Delta)+0(1) Q(\Delta) .
$$

The following theorem on wave interaction and cancellation is due to Glimm [1] and Glimm-Lax [2].

Theorem 2.5. Suppose that the initial data (1.2) has sufficiently small total variation T.V. Then

(i) total variation $\left\{u_{r}(\cdot, t) \mid-\infty<x<\infty\right\} \leqq 2 \mathrm{~T}$.V.

(ii) $Q \equiv \sum_{\Delta} Q(\Delta) \leqq 2(\mathrm{~T} . \mathrm{V} .)^{2}$.

(iii) $C=\sum_{\Delta} C(\Delta) \leqq \mathrm{T} . \mathrm{V} .+Q$.

Proof. Given any $I$-curve $J$, we set

$$
\begin{aligned}
& F(J)=L(J)+K Q(J), \\
& Q(J)=\sum\{|a b| \mid a \text { and } b \text { are strengths of interacting waves crossing } J\}, \\
& L(J)=\sum\{|a| \mid a \text { is the strengths of waves crossing } J\},
\end{aligned}
$$

where $K$ is a large constant. For any $I$-curves $J_{1}$ and $J_{2}, J_{2}$ an immediate successor $J_{1}$, it follows from Theorem 2.1 that

$$
\begin{aligned}
F\left(J_{2}\right)-F\left(J_{1}\right) & \leqq-K Q(\Delta)+0(1) Q(\Delta)+0(1) K L\left(J_{1}\right) Q(\Delta) \\
& \leqq\left[-K+0(1)+0(1) K L\left(J_{1}\right)\right] Q(\Delta),
\end{aligned}
$$

where $\Delta$ is the diamond between $J_{1}$ and $J_{2}$. Thus by choosing $K$ large we see that $F\left(J_{2}\right) \leqq F\left(J_{1}\right)$ provided that $F\left(J_{1}\right)$ is small. This argument implies inductively that if T.V. is small then $F(J)$ is small for all $J$ and (i) holds. Similarly, we have

$$
Q\left(J_{2}\right)-Q\left(J_{1}\right) \leqq-Q(\Delta)+0(1) L\left(J_{1}\right) Q(\Delta)
$$

which is less than $-\frac{1}{2} Q(\Delta)$ if T.V. is small. Summing the above inequality over all $\Delta$, we have

$$
Q(0) \geqq \sum_{J_{1}, J_{2}}\left[Q\left(J_{1}\right)-Q\left(J_{2}\right)\right] \geqq \sum_{\Delta} \frac{1}{2} Q(\Delta) \equiv \frac{1}{2} Q,
$$

where 0 is the $I$-curve in the zone $0 \leqq t \leqq s$. This proves (ii). Finally, estimate (iii) follows from (2.7) by summing over all $\Delta$. This completes the proof of the theorem. Q.E.D. 


\section{The Main Theorem}

In what follows $\left\{a_{m}\right\}$ is an equidistributed sequence in $(-1,1) . N=\frac{2}{\varepsilon}$ and $M$ are large integers. We divide the interval $(-1,1)$ into $N$ subintervals with equal length $\varepsilon$. Let $\left\{I_{i}\right\}_{i=1}^{2^{N}}$ be the collection of unions of any such subintervals. We set [cf. (1.7)]

$$
\delta=\sup _{\substack{1 \leqq p \leqq N \\ i=1, \ldots, 2^{N}}} \frac{B\left(a_{m-(p-1) M}, M, I_{i}\right)}{M}-\frac{\mu\left(I_{i}\right)}{2}
$$

which tends to zero as $M \rightarrow \infty$ for each fixed $N$.

Let $\varphi(x, t)$ be a test function supported in $0 \leqq t \leqq T$. We choose $M$ so that

$(N-1) M s \leqq T \leqq N M s$.

It is noted that for each fixed $N$

$$
M \rightarrow \infty, \quad N M s \leqq 2 T, \text { and } M s \leqq T \varepsilon
$$

as the mesh length $s \rightarrow 0$. Since $\varphi$ is smooth and has compact support, we have

$$
\begin{aligned}
& \left|\varphi(x, t)-\varphi\left(x^{\prime}, t^{\prime}\right)\right|=0(\varepsilon) \\
& \text { if }\left|x-x^{\prime}\right|+\left|t-t^{\prime}\right|=0(\varepsilon), \text { as } \varepsilon \rightarrow 0 .
\end{aligned}
$$

To express the error term (1.6) in terms of elementary waves, we will partition the elementary waves in the following way. Suppose that elementary $k$-waves $\left(u_{k-1}, u_{k}\right)$, $k=1,2, \ldots, n$, issue from $(i r, j s), i+j=$ even. If $\left(u_{k-1}, u_{k}\right)$ is a $k$-shock wave, then we choose any vectors $y_{0}, y_{1}, \ldots, y_{l}, y_{0}=u_{k-1}, y_{l}=u_{k}, y_{h} \in S_{k}^{-}\left(u_{k-1}\right), \lambda_{k}\left(y_{h}\right)<\lambda_{k}\left(y_{h-1}\right)$, $h=1,2, \ldots, l$, and set

$$
\begin{aligned}
& v_{k}^{h}(i, j)=y_{h}-y_{h-1} . \\
& \lambda_{k}^{h}(i, j)=\sigma\left(u_{k-1}, u_{k}\right) .
\end{aligned}
$$

If $\left(u_{k-1}, u_{k}\right)$ is a $k$-rarefaction wave, we choose vectors $y_{0}, y_{1}, \ldots, y_{l}, y_{0}=u_{k-1}, y_{l}=u_{k}$, $y_{h} \in R_{k}^{+}\left(u_{k-1}\right), \lambda_{k}\left(y_{h}\right)>\lambda_{k}\left(y_{h-1}\right), h=1,2, \ldots, l$, and set

$$
\begin{aligned}
& v_{k}^{h}(i, j)=y_{h}-y_{h-1}, \\
& \lambda_{k}^{h}(i, j)=\lambda_{k}\left(y_{h-1}\right) .
\end{aligned}
$$

In the second case we require that

$$
\left|\lambda_{k}\left(y_{h}\right)-\lambda_{k}\left(y_{h-1}\right)\right| \leqq \varepsilon, \quad h=1,2, \ldots, l,
$$

and to make sure that $\left\{v_{k}^{h}(i, j)\right\}$ is not partitioned further at $t=(j+1) s$, we also require that

$$
a_{j+1} \notin\left(\lambda_{k}\left(y_{h-1}\right), \lambda_{k}\left(y_{h}\right)\right), \quad h=1,2, \ldots, l .
$$

In what follows, for brevity, we will write the approximate solutions as $u_{r}(x, t)$ and the error term (1.6) $E_{j}\left(r, \varphi, a_{n}\right)$ as $E(j, r)$ etc. It follows from (3.3) (3.7) that

$$
\begin{aligned}
E(j, r)= & \sum_{i, h, k}\left[\lambda_{k}^{h}(i, j) s+r \operatorname{sign}\left(r a_{j+1}-\lambda_{k}^{h}(i, j) s\right)\right] v_{k}^{h}(i, j) \varphi(i r, j s) \\
& +0(1)[\varepsilon+s] s \sum_{i, h, k}\left\|v_{k}^{h}(i, j)\right\| \\
= & \sum_{i, h, k}\left[\lambda_{k}^{h}(i, j) s+r \operatorname{sign}\left(r a_{j+1}-\lambda_{k}^{h}(i, j) s\right)\right] v_{k}^{h}(i, j) \varphi(i r, j s)+0(1) \varepsilon s \mathrm{~T} . \mathrm{V} .,
\end{aligned}
$$


where T.V. is the total variation of the initial data (cf. Theorem 2.5), and

$$
\operatorname{sign} x=\left\{\begin{array}{rll}
1 & \text { if } & x>0 \\
-1 & \text { if } & x \leqq 0
\end{array}\right.
$$

Theorem 3.1. Suppose that the total variation T.V. of the initial data (1.2) is small. Then for any equidistrubted sequence $\left\{a_{m}\right\}$ in $(-1,1)$, the Glimm scheme converges to yield an exact solution of (1.1) and (1.2).

The proof of the theorem depends on the following lemma which states that elementary waves can be partitioned so that their speeds and strengths can be traced.

Lemma 3.2. There exists a partition of elementary waves $\left\{v_{k}^{h}(i, j), \lambda_{k}^{h}(i, j)\right\}$ which satisfies (3.4) (3.7) and, moreover, $\left\{v_{k}^{h}(i, j), \lambda_{k}^{h}(i, j)\right\}$ is a disjoint union of $\left\{\tilde{v}_{k}^{h}(i, j)\right.$, $\left.\tilde{\lambda}_{k}^{h}(i, j)\right\}$ and $\left\{\tilde{v}_{k}^{h}(i, j), \tilde{\lambda}_{k}^{h}(i, j)\right\}$, so that for any $j,(p-1) M \leqq j \leqq p M, p \in\{1,2, \ldots, N\}$,

(i) $\sum_{i, h, k} M \tilde{v}_{k}^{h}(i, j) \| \leqq 0(1)\left[Q\left(\Lambda_{p}\right)+C\left(\Lambda_{p}\right)\right]$,

and there is a one-to-one correspondence between $\left\{\tilde{v}_{k}^{h}(i, j), \tilde{\lambda}_{k}^{h}(i, j)\right\}$ and $\left\{\tilde{v}_{k}^{h}(i,(p-1) M)\right.$, $\tilde{\lambda}_{k}^{h}(i,(p-1) M\}$ :

$$
\left(\tilde{v}_{k}^{h}(i,(p-1) M), \tilde{\lambda}_{k}^{h}(i,(p-1) M)\right) \leftrightarrow\left(\tilde{v}_{k}^{h}\left(i_{j}, j\right), \tilde{\lambda}_{k}^{h}\left(i_{j}, j\right)\right)
$$

such that

(ii) $\sum_{i, h, k}\left\|\tilde{v}_{k}^{h}(i,(p-1) M)-\tilde{v}_{k}^{h}\left(i_{j}, j\right)\right\|=0(1) Q\left(\Lambda_{p}\right)$,

(iii) $\sum_{i, h, k}\left\|\tilde{v}_{k}^{h}(i,(p-1) M)\right\| \cdot \max _{(p-1) M \leqq j \leqq p M}\left|\tilde{\lambda}_{k}^{h}\left(i_{j}, j\right)-\tilde{\lambda}_{k}^{h}(i,(p-1) M)\right|=0(1) Q\left(\Lambda_{p}\right)$,

(iv) $\left|i-i_{j}\right| \leqq|j-(p-1) M|$.

Here the bounds $0(1)$ are independent of $i, j, r$, and $\Lambda_{p}$ is the zone $(p-1) M s \leqq t \leqq p M s$.

The above lemma, whose proof we will postpone until later, will now be used to prove Theorem 3.1.

Proof of Theorem 3.1. It follows from (3.8) and (i), (ii) of Lemma 3.2 that for any $j$, $(p-1) M \leqq j \leqq p M$,

$$
\begin{aligned}
E(j, r)= & \sum_{i, h, k}\left[\tilde{\lambda}_{k}^{h}(i, j) s+r \operatorname{sign}\left(r a_{j+1}-\tilde{\lambda}_{k}^{h}(i, j) s\right)\right] \tilde{v}_{k}^{h}(i, j) \varphi(i r, j s) \\
& +0(1)\left(\varepsilon s \mathrm{~T} . \mathrm{V} .+r Q\left(\Lambda_{p}\right)+r C\left(\Lambda_{p}\right)\right) \\
= & \sum_{i, h, k}\left[\tilde{\lambda}_{k}^{h}(i,(p-1) M) s+r \operatorname{sign}\left(r a_{j+1}\right.\right. \\
& \left.\left.-\tilde{\lambda}_{k}^{h}\left(i_{j}, j\right) s\right)\right] \tilde{v}_{k}^{h}(i,(p-1) M) \varphi\left(i_{j} r, j s\right) \\
& +0(1)\left(\varepsilon s \mathrm{~T} . \mathrm{V} .+r Q\left(\Lambda_{p}\right)+r C\left(\Lambda_{p}\right)\right) .
\end{aligned}
$$


Because of the domain of dependency condition (iv), Lemma 3.2 and the smoothness of $\varphi,(3.3)$, we have

$$
\varphi\left(i_{j} r, j s\right)=\varphi(i r,(p-1) M s)+0(1)(M s) .
$$

We note that sign $\left(r a_{j+1}-\tilde{\lambda}_{k}^{h}\left(i_{j}, j\right) s\right)$ differs from sign $\left(r a_{j+1}-\tilde{\lambda}_{k}^{h}(i,(p-1) M) s\right)$ only if $a_{j+1}$ lies between $\tilde{\lambda}_{k}^{h}\left(i_{j}, j\right) s / r$ and $\tilde{\lambda}_{k}^{h}(i,(p-1) M) s / r$. Thus it follows from (3.1) and (iii), Lemma 3.2 that

$$
\begin{aligned}
& \sum_{j=(p-1) M}^{p M} \sum_{i, h, k} \operatorname{sign}\left(r a_{j+1}-\tilde{\lambda}_{k}^{h}\left(i_{j}, j\right) s\right) \tilde{v}_{k}^{h}(i,(p-1) M) \varphi\left(i_{j} r, j s\right) \\
= & \sum_{j=(p-1) M}^{p M} \sum_{i, h, k} \operatorname{sign}\left(r a_{j+1}-\tilde{\lambda}_{k}(i,(p-1) M) s\right) \tilde{v}_{k}^{h}(i,(p-1) M) \varphi\left(i_{j} r, j s\right) \\
& +0(1) \sum_{i, h, k} B\left(a_{m-(p-1) M}, M, I_{i, h, k}\right) \tilde{v}_{k}^{h}(i,(p-1) M) \\
= & \sum_{j=(p-1) M}^{p M} \sum_{i, h, k} \operatorname{sign}\left(r a_{j+1}-\tilde{\lambda}_{k}(i,(p-1) M) s\right) \tilde{v}_{k}^{h}(i,(p-1) M) \varphi\left(i_{j} r, j s\right) \\
& +0(1) M\left[\sum_{i, h, k} \max _{(p-1) M \leqq j \leqq p M}\left|\tilde{\lambda}_{k}^{h}\left(i_{j}, j\right)-\tilde{\lambda}_{k}^{h}(i,(p-1) M)\right|\right. \\
= & \quad \sum_{j=(p-1) M}^{p M} \sum_{i, h, k} \operatorname{sign}\left(r a_{j+1}-\tilde{\lambda}_{k}^{h}(i,(p-1) M) s\right) \tilde{v}_{k}^{h}(i,(p-1) M) \varphi\left(i_{j} r, j s\right) \\
& +0(1) M(2 \varepsilon+\delta) \mathrm{T} . \mathrm{V} .+0(1) M Q\left(\Lambda_{p}\right),
\end{aligned}
$$

where $I_{i, h, k}=\left(\min _{(p-1) M \leqq j \leqq p M} \tilde{j}_{k}^{h}\left(i_{j}, j\right), \max _{(p-1) M \leqq j \leqq p M} \tilde{\lambda}_{k}^{h}\left(i_{j}, j\right)\right)$.

We have from $(3.8) \sim(3.10)$,

$$
\begin{aligned}
\sum_{j=(p-1) M}^{p M} E(j, r) & \\
= & \sum_{i, h, k}\left[M \tilde{\lambda}_{k}^{h}(i,(p-1) M) s+r \sum_{j=(p-1) M} \operatorname{sign}\left(r a_{j+1}-\tilde{\lambda}_{k}^{h}(i,(p-1) M) s\right]\right. \\
& \cdot \tilde{v}_{k}^{h}(i,(p-1) M) \varphi(i,(p-1) M) \\
& +0(1) M\left[r s \mathrm{~T} . \mathrm{V} .+r Q\left(\Lambda_{p}\right)+r C\left(\Lambda_{p}\right)+\varepsilon r \mathrm{~T} . \mathrm{V} .+\delta r \mathrm{~T} . \mathrm{V} .\right] .
\end{aligned}
$$

It follows easily from (3.1) that for any $y$

$$
M y s+r \sum_{j=(p-1) M}^{p M} \operatorname{sign}\left(r a_{j+1}-y s\right) \leqq M(\delta+\varepsilon) r,
$$

and thus we conclude from (3.11)

$$
\sum_{j=(p-1) M}^{p M} E(j, r)=0(1) \operatorname{Mr}\left[(\delta+\varepsilon) \text { T.V. }+Q\left(\Lambda_{p}\right)+C\left(\Lambda_{p}\right)\right] .
$$


Summing (3.13) over $p=1,2, \ldots, N$, we finally obtain

$$
E\left(\varphi, a_{m}\right)=0(1) T[(\delta+\varepsilon) \text { T.V. }+r Q+r C],
$$

where we have used (3.2) and the following inequality

$$
\sum_{p=1}^{N}\left(Q\left(\Lambda_{p}\right)+C\left(\Lambda_{p}\right)\right) \leqq Q+C,
$$

which is bounded, by Theorem 2.5. Since $\delta$ and $r$ approach zero as $M \rightarrow \infty$ for each fixed $\varepsilon$, and $\varepsilon$ can be chosen arbitrarily, we conclude that $E\left(\varphi, a_{m}\right)$ goes to zero as $r$ goes to zero. This completes the proof of the theorem. Q.E.D.

Proof of Lemma 3.2. For each fixed $p \in\{1,2, \ldots, N\}$, the sequence $\left\{v_{k}^{h}(i, j), \lambda_{k}^{h}(i, j)\right\}$, $(p-1) M \leqq j \leqq p M$, is defined as follows: First, we will construct inductively the sequences $\left\{v_{k}^{h}\left(i, j^{\prime}, j\right), \lambda_{k}^{h}\left(i, j^{\prime}, j\right)\right\},(p-1) M \leqq j^{\prime} \leqq j \leqq p M$, which satisfy (3.4) (3.7) and, moreover, $\tilde{\lambda}$ the sequence is a disjoint union of $\left\{\tilde{v}_{k}^{h}\left(i, j^{\prime}, j\right), \tilde{\lambda}_{k}^{h}\left(i, j^{\prime}, j\right)\right\}$ and $\left\{\tilde{\tilde{v}}_{k}^{h}\left(i, j^{\prime}, j\right), \tilde{\tilde{\lambda}}_{k}^{h}\left(i, j^{\prime}, j\right)\right\}$ such that for $(p-1) M \leqq j^{\prime} \leqq j \leqq p M$

$$
\begin{aligned}
& \sum_{i, h, k}\left\|\tilde{\tilde{v}}_{k}^{h}\left(i, j^{\prime}, j\right)\right\| \\
& =0(1)[Q((p-1) M s \leqq t \leqq j s)+C((p-1) M s \leqq t \leqq j s)],
\end{aligned}
$$

and there is an one-to-one correspondence

$$
\tilde{v}_{k}^{h}\left(i_{j^{\prime}}, j^{\prime}, j\right) \leftrightarrow \tilde{v}_{k}^{h}(i,(p-1) M, j), \quad(p-1) M \leqq j^{\prime} \leqq j,
$$

such that

$$
\begin{aligned}
& \sum_{i, h, k}\left\|\tilde{v}_{k}^{h}\left(i_{j^{\prime}}, j^{\prime}, j\right)-\tilde{v}_{k}^{h}(i,(p-1) M, j)\right\| \\
& =0(1) Q\left((p-1) M s \leqq t \leqq j^{\prime} s\right), \\
& \left|i_{j^{\prime}}-i\right| \leqq\left|j^{\prime}-(p-1) M\right|, \\
& \sum_{i, h, k}\left|\tilde{\lambda}_{k}^{h}\left(i_{j^{\prime}}, j^{\prime}, j\right)-\tilde{\lambda}_{k}^{h}(i,(p-1) M, j)\right|\left\|\tilde{v}_{k}^{h}(i,(p-1) M, j)\right\| \\
& =0(1) Q\left((p-1) M s \leqq t \leqq j^{\prime} s\right) .
\end{aligned}
$$

To prove the lemma, we simply set $\left\{v_{k}^{h}(i, j), \lambda_{k}^{h}(i, j)\right\},(p-1) M \leqq j \leqq p M$, to be $\left.\left\{v_{k}^{h}(i, j, p M), \lambda_{k}^{h}(i, j), p M\right)\right\}$ and (i) (iv) follows from (3.15) (3.18).

We now establish $(3.15) \sim(3.18)$ by induction on $j$. For $j=(p-1) M$, we let $\left\{v_{k}^{h}(i,(p-1) M,(p-1) M), \lambda_{k}^{h}(i,(p-1) M,(p-1) M)\right\}$ be any partition satisfying (3.4) (3.7). Suppose that $\left\{v_{k}^{h}\left(i, j^{\prime}, j\right), \lambda_{k}^{h}\left(i, j^{\prime}, j\right)\right\}$ has been constructed for $(p-1) M \leqq j^{\prime}$ $\leqq j \leqq p M$. We have to construct $\left\{v_{k}^{h}\left(i, j^{\prime}, j+1, \lambda_{k}^{h}\left(i, j^{\prime}, j+1\right)\right\}\right.$ for $(p-1) M \leqq j^{\prime} \leqq j+1$. For a given point $(i r,(j+1) s)$ let $\left(w_{k-1}, w_{k}\right), k=1,2, \ldots, N$ be the elementary $k$-wave issued from the point. Suppose that these waves are produced by the interaction in the diamond $\Delta$ of waves $\left(u_{k-1}, u_{k}\right), k=1,2, \ldots, n$, issued from $((i-1) r, j s)$ and $\left(v_{k-1}, v_{k}\right), k=1,2, \ldots, n$ issued from $((i+1) r, j s)$. Since $\left\{v_{k}^{h}(i, j, j), \lambda_{k}^{h}(i, j, j)\right\}$ satisfies (3.7), there exist points $\tilde{y}_{0}, \tilde{y}_{1}, \ldots, \tilde{y}_{h_{1}}$ on $S_{k}^{-}\left(u_{k-1}\right) \cup R_{k}^{+}\left(u_{k-1}\right)$ and $\tilde{y}_{0}, \tilde{\tilde{y}}_{1}, \ldots, \tilde{\tilde{y}}_{h_{2}}$ on $S_{k}^{-}\left(v_{k-1}\right) \cup R_{k}^{+}\left(v_{k-1}, \tilde{y}_{0}=u_{k-1}, \tilde{y}_{h_{1}}=u_{k}, \tilde{y}_{0}=v_{k-1}, \tilde{\tilde{y}}_{h_{2}}=v_{k}\right.$, such that after some renumberings,

$$
\begin{array}{ll}
\tilde{y}_{h}-\tilde{y}_{h-1}=v_{k}^{h}(i-1, j, j), & h=1,2, \ldots, h_{1}, \\
\tilde{y}_{h}-\tilde{y}_{h-1}=v_{k}^{h}(i+1, j, j), & h=1,2, \ldots, h_{2} .
\end{array}
$$


We now construct the sequence $\left\{v_{k}^{h}\left(i, j^{\prime}, j+1\right), \lambda_{k}^{h}\left(i, j^{\prime}, j+1\right)\right\}$ according to the various cases in Theorem 2.3.

Suppose that both $\left(u_{k-1}, u_{k}\right)$ and $\left(v_{k-1}, v_{k}\right)$ are shock waves and $\left(w_{k-1}, w_{k}\right)$ is a rarefaction wave. Then it follows from Theorem 2.2 that

$$
\left\|u_{k-1}-u_{k}\right\|+\left\|v_{k-1}-v_{k}\right\|+\left\|w_{k-1}-w_{k}\right\|=0(1) Q(\Delta) .
$$

In this case we simply partition $\left(w_{k-1}, w_{k}\right)$ according (3.4) (3.7) and classify $\left\{v_{k}^{h(i, j+1, j+1)\}}\right.$ as $\left\{\tilde{v}_{k}^{h}(i j+1, j+1)\right\}$. Furthermore, we classify all waves $\left\{\tilde{v}_{k}^{h}\left(i_{j^{\prime}}^{\prime}, j^{\prime}, j\right)\right\},(p-1) M \leqq j^{\prime} \leqq j$ relating to $\left.\left\{v_{k}^{h} i-1, j, j\right)\right\}_{h=1}^{h_{1}},\left\{v_{k}^{h}(i+1, j, j)\right\}_{h=1}^{h_{2}}$ :

$$
\tilde{v}_{k}^{h}(i \pm 1, j, j) \leftrightarrow \tilde{v}_{k}^{h}\left(i^{\prime},(p-1) M, j\right) \leftrightarrow \tilde{v}_{k}^{h}\left(i_{j^{\prime}}^{\prime}, j^{\prime}, j\right)
$$

as members of $\left\{\tilde{v}_{k}^{h}\left(i_{j^{\prime}}^{\prime}, j^{\prime}, j+1\right)\right\}$. We note that because of (3.19) the total amount of waves reclassified is $0(1) Q(\Delta)$ plus

$$
\sum_{h}\left\|\tilde{v}_{k}^{h}\left(i_{j^{\prime}}^{\prime}, j^{\prime}, j\right)-\tilde{v}_{k}^{h}\left(i^{\prime},(p-1) M, j\right)\right\|
$$

and thus (ii) of the lemma still holds for $\left\{v_{k}^{h}\left(i, j^{\prime}, j+1\right), \lambda_{k}^{h}\left(i, j^{\prime}, j+1\right)\right\}$. Other statements of the lemma holds trivially.

We next suppose that $\left(u_{k-1}, u_{k}\right),\left(v_{k-1}, v_{k}\right),\left(w_{k-1}, w_{k}\right)$ are shock waves. According to Theorem 2.2, there are three cases: (a) $0 \leqq \lambda_{k}\left(w_{k-1}\right)-\lambda_{k}\left(w_{k}\right)-\left(\lambda_{k}\left(u_{k-1}\right)\right.$ $-\lambda_{k}\left(u_{k}\right)+\lambda_{k}\left(u_{k-1}\right)-\lambda_{k}\left(v_{k}\right)=0(1) Q(\Delta) ; \quad$ (b) $0 \leqq \lambda_{k}\left(u_{k-1}\right)-\lambda_{k}\left(u_{k}\right)-\left(\lambda_{k}\left(w_{k-1}\right)-\lambda_{k}\left(w_{k}\right)\right)$ $=0(1) Q(\Delta), \quad \lambda_{k}\left(v_{k-1}\right)-\lambda_{k}\left(v_{k}\right)=0(1) Q(\Delta) ;$ (c) $0 \leqq \lambda_{k}\left(u_{k-1}\right)-\lambda_{k}\left(u_{k}\right)+\lambda_{k}\left(v_{k-1}\right)-\lambda_{k}\left(v_{k}\right)$ $-\left(\lambda_{k}\left(w_{k-1}\right)-\lambda_{k}\left(w_{k}\right)\right)=0(1) Q(\Delta)$. In Case (a) we choose $y_{h} \in S_{k}^{-}\left(w_{k-1}\right)$, $h=0,1,2, \ldots, h_{1}+h_{2}, y_{0}=w_{k-1}, \lambda_{k}\left(y_{h}\right)-\lambda_{k}\left(y_{0}\right)=\lambda_{k}\left(\tilde{y}_{h}\right)-\lambda_{k}\left(\tilde{y}_{0}\right)$ for $h=1,2, \ldots, h_{1}$, $\lambda_{k}\left(y_{h}\right)-\lambda_{k}\left(y_{h_{1}}\right)=\lambda_{k}\left(\tilde{\tilde{y}}_{h-h_{1}}\right)-\lambda_{k}\left(\tilde{\tilde{y}}_{0}\right)$ for $h=1,2, \ldots, h_{2}$, and $y_{h}-y_{h-1} \leftrightarrow \tilde{y}_{h}-\tilde{y}_{h-1}$ for $h=1,2, \ldots, h_{1}, y_{h+h_{2}}-y_{h+h_{1-1}} \leftrightarrow \tilde{y}_{h}-\tilde{y}_{h-1}$ for $h=1,2, \ldots, h_{2}$. We note that if $\tilde{y}_{h}$ $-\tilde{y}_{j-1} \leftrightarrow \tilde{v}_{k}^{h}\left(i_{0},(p-1) M, j\right)$ then $i_{j+1}=i_{0}$ and $\left|i-i_{0}\right| \leqq\left|i-1-i_{0}\right|+1$ which, by induction hypothesis, is less than $|j-(p-1) M|+1 \leqq|j+1-(p-1) M|$ and so (iv) of the lemma holds. It follows from (i), Theorem 2.3 that

$$
\begin{aligned}
& \sum_{h=1}^{h_{1}}\left|\sigma\left(w_{k-1}, w_{k}\right)-\sigma\left(u_{k-1}, u_{k}\right)\right|\left\|\tilde{y}_{h}-\tilde{y}_{h-1}\right\| \\
& \leqq 2\left|\sigma\left(w_{k-1}, w_{k}\right)-\sigma\left(u_{k-1}, u_{k}\right)\right|\left\|u_{k-1}-u_{k}\right\|=0(1) Q(\Delta)
\end{aligned}
$$

and thus (iii) of the lemma holds. Finally, we classify $v_{k}^{h_{1}+h_{2}+1}(i, j+1)=w_{k}-y_{h_{1}+h_{2}}$ $=0(1) Q(\Delta)$ as a member of $\left\{\tilde{v}_{k}^{h}(i, j+1)\right\}$. We now turn to Case (b). We choose $y_{h} \in S_{k}^{-}\left(w_{k-1}\right), h=0,1, \ldots, h_{3}, 0 \leqq h_{3} \leqq h_{1}$, and choose $\tilde{y} \in S_{k}^{-}\left(u_{k-1}\right)$ between $\tilde{y}_{h_{3}-1}$ and $\tilde{y}_{h_{3}}, y_{0}=w_{k-1}, \lambda_{k}\left(y_{h}\right)-\lambda_{k}\left(y_{0}\right)=\lambda_{k}\left(\tilde{y}_{h}\right)-\lambda_{k}\left(\tilde{y}_{0}\right)$ for $h=1,2, \ldots, h_{3}-1$, and $0 \leqq \lambda_{k}\left(y_{h_{3}-1}\right)$ $-\lambda_{k}\left(y_{h_{3}}\right)=\lambda_{k}(\tilde{y})-\lambda_{k}\left(\tilde{y}_{h_{3}-1}\right)$. We first refine the partition of $\left(u_{k-1}, u_{k}\right)$ with the addition of the dividing point $\tilde{y}$. We also refine the vectors $\tilde{v}_{k}^{h}\left(i^{\prime}, j^{\prime}, j\right),(p-1) M \leqq j^{\prime} \leqq j$, which are related to $y_{h_{3}}-y_{h_{3}-1}$ :

$$
y_{h_{3}}-y_{h_{3}-1} \leftrightarrow \tilde{v}_{k}^{h}\left(i_{0},(p-1) M, j\right) \leftrightarrow \tilde{v}_{k}^{h}\left(i^{\prime}, j^{\prime}, j\right)
$$

in a similar way. We note that when a partition is refined, condition (ii) $\sim$ (iv) of the lemma still hold because of (ii), Lemma 2.4 . 
The vectors $y_{h}-y_{h-1}, h=1,2, \ldots, \mathrm{h}-1$, and $y_{h_{3}}-y_{h_{3}-1}$ are then related to $\tilde{y}_{h}-\tilde{y}_{h-1}$ and $\tilde{y}-\tilde{y}_{h_{3}-1}$ respectively. Finally, the vectors $\tilde{y}_{h_{3}}-\tilde{y}, \tilde{y}_{h}-\tilde{y}_{h-1}$, $h=h_{3}+1, \ldots, h_{1}$, and $\tilde{y}_{h}-\tilde{y}_{h-1}, h=1,2, \ldots, h_{2}$ are reclassified as members of $\left\{\tilde{\tilde{v}}_{k}^{h}(i \pm 1, j, j+1)\right\}$ no matter how they were classified in $\left\{v_{k}^{h}(i \pm 1, j, j)\right\}$. The total amount of waves reclassified is $0(1) Q(\Delta)$, Theorem 2.2. Case (c) is dealt with in a similar way. Details are omitted.

We now consider the case where $\left(u_{k-1}, u_{k}\right)$ is a shock wave, and $\left(v_{k-k}, v_{k}\right)$ and $\left(w_{k-1}, w_{k}\right)$ are rarefaction waves. There are two cases: (a) $\lambda_{k}\left(w_{k}\right)-\lambda_{k}\left(w_{k-1}\right) \leqq \lambda_{k}\left(v_{k}\right)$ $-\lambda_{k}\left(v_{k-1}\right)$ and (b) $\lambda_{k}\left(w_{k}\right)-\lambda_{k}\left(w_{k-1}\right)>\lambda_{k}\left(v_{k}\right)-\lambda_{k}\left(v_{k-1}\right)$. For brevity, we only treat Case (a). Thus there exists $\tilde{\tilde{y}} \in R_{k}^{+}\left(v_{k-1}\right)$ between $\tilde{\tilde{y}}_{h_{3}-1}$ and $\tilde{\tilde{y}}_{h_{3}}, 1 \leqq h_{3} \leqq h_{2}$, with $\lambda_{k}(\tilde{y})$ $-\lambda_{k}\left(v_{k}\right)=\lambda_{k}\left(w_{k-1}\right)-\lambda_{k}\left(w_{k}\right)$. If $a_{j+1}$ lies between $\lambda_{k}\left(w_{k-1}\right)$ and $\lambda_{k}\left(w_{k}\right)$, we choose $\tilde{y}^{\prime} \in R_{k}^{+}\left(v_{k-1}\right)$ such that $\lambda_{k}\left(\tilde{y}^{\prime}\right)-\lambda_{k}\left(v_{k}\right)=a_{j+1} r-\lambda_{k}\left(w_{k}\right)$. We first refine the partition of $\left(v_{k-1}, v_{k}\right)$ by the additional dividing points $\tilde{\tilde{y}}$ and $\tilde{\tilde{y}}^{\prime}$. For simplicity, we denote this refined partition as $\left\{\tilde{\tilde{y}}_{h}-\tilde{y}_{h-1}\right\}, h=1,2, \ldots, \mathrm{h}_{2}$ with $\lambda_{k}\left(\tilde{y}_{h_{3}}\right)-\lambda_{k}\left(v_{k}\right)=\lambda_{k}\left(w_{k-1}\right)$ $-\lambda_{k}\left(w_{k}\right)$. The partition $\left\{v_{k}^{h}\left(i^{\prime}, j^{\prime}, j\right)\right\},(p-1) M \leqq j^{\prime} \leqq j$, which is related to $\left\{v_{k}^{h}(i+1, j, j)\right\}$, is also refined in an obvious way. We now partition $\left(w_{k-1}, w_{k}\right)$ by choosing $y_{h} \in R_{k}^{+}\left(w_{k-1}\right), h=0,1,2, \ldots, h_{2}-h_{3}, \lambda_{k}\left(y_{h}\right)-\lambda_{k}\left(w_{k}\right)=\lambda_{k}\left(\tilde{y}_{h+h_{3}}\right)-\lambda_{k}\left(v_{k}\right)$. As we see, the dividing point, $\tilde{\tilde{y}}^{\prime}$ was added in the partition of $\left(v_{k-1}, v_{k}\right)$ so that (3.7) holds for $\left\{v_{k}^{h}(i, j+1, j+1)\right\}$. To check (iii) of the lemma, we see from (iii), Theorem 2.3 and (i) Lemma 2.4 that

$$
\begin{aligned}
& \sum_{h=0}^{h_{2}-h_{3}}\left|\lambda_{k}\left(y_{h}\right)-\lambda_{k}\left(\tilde{y}_{h+h_{3}}\right)\right|\left\|y_{h}-y_{h+1}\right\| \\
& =\left|\lambda_{k}\left(w_{k}\right)-\lambda_{k}\left(v_{k}\right)\right| \sum_{h=0}^{h_{2}-h_{3}}\left\|y_{h}-y_{h+1}\right\| \\
& \leqq 2\left|\lambda_{k}\left(w_{k}\right)-\lambda_{k}\left(v_{k}\right)\right|\left\|w_{k}-w_{k-1}\right\| \\
& =0(1) Q(\Delta) .
\end{aligned}
$$

Other parts of the lemma are proved using Theorem $2.1 \sim 2.3$ and the induction hypothesis. Details are omitted. Finally, the waves in $\left\{v_{k}^{h}(i \pm 1, j, j)\right\}$ which partition $\left(u_{k-1}, u_{k}\right)$ and $\left(v_{k-1}, \tilde{\tilde{y}}_{h_{3}}\right)$ along with those related wave in $\left\{v_{k}^{h}\left(i^{\prime}, j^{\prime}, j\right)\right\}$ are reclassified as members of $\left\{\tilde{\hat{v}}_{k}^{h}(i \pm 1, j, j+1)\right\}$ and $\left\{\tilde{v}_{k}^{h}\left(i^{\prime}, j^{\prime}, j+1\right)\right\}$, respectively. The amount of waves so reclassified is $2 C(\Delta)$, Theorem 2.2 , so that (ii) of the lemma holds.

Other waves interaction (cf. Theorem 2.3) are treated in a similar way. This completes the proof of the lemma. Q.E.D.

Remark. The rate of convergence depends on how well distributed the sequence $\left\{a_{m}\right\}$ is in $(-1,1)$, [cf. (3.14)]. It seems that our method does not yield optimal rate of convergence. The rate is always less than first order, and in general, is algebraic.

\section{References}

1. Glimm, J.: Solutions in the large for nonlinear hyperbolic systems of equations. Commun. Pure Appl. Math. 18, 697-715 (1965)

2. Glimm,J., Lax, P.D.: Decay of solutions of systems of nonlinear hyperbolic conservation laws. Amer. Math. Soc. $101(1970)$ 
3. Lax, P.D.: Hyperbolic systems of conservation laws. II. Commun. Pure Appl. Math. 10, 537-566 (1957)

4. Liu,T.-P.: The Riemann problem for general systems of conservation laws. J. Diff. Equations 18, $218-234(1975)$

5. Liu, T.-P.: Large-time behavior of solutions of initial and initial-boundary value problem of general system of hyperbolic conservation laws. Commun. math. Phys. 55, 163-177 (1977)

6. Lax,P.D.: Hyperbolic systems of conservation laws and the mathematical theory of shock waves. Philadelphia SIAM Regional Conf. Ser. in Appl. Math. 11 (1973)

Communicated by J. Glimm

Received June 20, 1977 